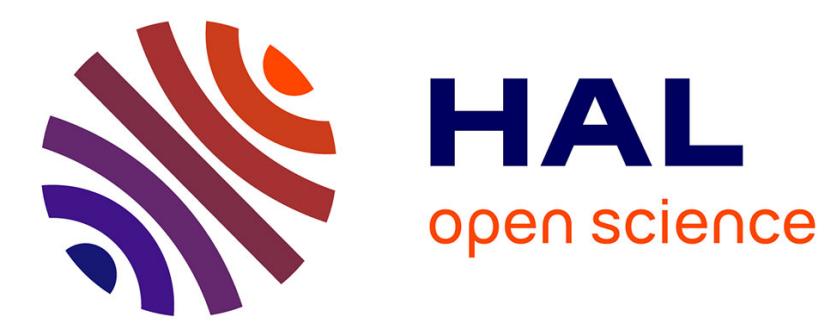

\title{
Ramifications discrètes de routes marchandes transnationales : circulation de la pacotille chinoise entre marchés ruraux et villes secondaires au Cameroun Sylvain Racaud
}

\section{- To cite this version:}

Sylvain Racaud. Ramifications discrètes de routes marchandes transnationales : circulation de la pacotille chinoise entre marchés ruraux et villes secondaires au Cameroun. Les Cahiers d'Outre-Mer. Revue de géographie de Bordeaux, 2018, 71 (278), pp.433-464. 10.4000/com.9441 . hal-02610604

\author{
HAL Id: hal-02610604 \\ https://hal.science/hal-02610604
}

Submitted on 13 Jun 2020

HAL is a multi-disciplinary open access archive for the deposit and dissemination of scientific research documents, whether they are published or not. The documents may come from teaching and research institutions in France or abroad, or from public or private research centers.
L'archive ouverte pluridisciplinaire HAL, est destinée au dépôt et à la diffusion de documents scientifiques de niveau recherche, publiés ou non, émanant des établissements d'enseignement et de recherche français ou étrangers, des laboratoires publics ou privés. 


\title{
Ramifications discrètes de routes marchandes transnationales : circulation de la pacotille chinoise entre marchés ruraux et villes secondaires au Cameroun
}

\author{
Sylvain Racaud \\ Université Bordeaux-Montaigne, UMR 5115 LAM \\ Sylvain.racaud@u-bordeaux-montaigne.fr
}

\author{
« Les Cahiers d'Outre-Mer», 2018/2 n 278 | pages 433 à 464. \\ Référence électronique
}

Sylvain Racaud, «Ramifications discrètes de routes marchandes transnationales : circulation de la pacotille chinoise entre marchés ruraux et villes secondaires au Cameroun », Les Cahiers d'Outre-Mer [En ligne], 278 | Juillet-Décembre, mis en ligne le 01 janvier 2024, consulté le 12 février 2020. URL : http://journals.openedition.org/com/9441 ; DOI : 10.4000/com.9441

Résumé

Dans quelle mesure la circulation de la pacotille chinoise relie des marges rurales et des villes secondaires à des routes marchandes transnationales ? A partir d'entretiens réalisés avec des commerçants à Bafoussam et dans les monts Bamboutos (Ouest Cameroun) et d'observations de lieux marchands, cet article analyse des dynamiques spatiales liées à la circulation de produits bon marché importés de Chine. En prenant pour objet la route marchande des pacotilles chinoises, il montre comment des marges rurales et des villes secondaires - ramifications discrètes - s'intègrent à l'économie mondialisée via la circulation de la pacotille chinoise et comment ce mouvement contribue à des relations rural-urbain sans précédent. Le propos porte successivement sur l'objet «route marchande de la pacotille chinoise », sur les lieux marchands, sur les mobilités marchandes et enfin sur les logiques de réseau. L'article conclue en soulignant le rôle déterminant du secteur privé dans la structuration de cette route marchande dont les logiques de réseau dépassent le continuum urbain-rural.

Mots-clés : route marchande, pacotille chinoise, commerce bon marché, mobilité, réseau, urbain-rural, Cameroun, sino-africain

\section{Inconspicuous branches of transnational trade routes: circulation of Chinese junk goods between rural markets and secondary cities in Cameroon}

\section{Abstract}

To what extent does the circulation of Chinese junk traffic connect rural margins and secondary cities with transnational trade routes? Based on interviews with traders in Bafoussam and the Bamboutos Mountains (West Cameroon) and observations of trading places, this article analyses the spatial dynamics linked to the circulation of cheap products imported from China. By focusing on the Chinese junk trade route, it shows how rural margins and secondary cities - discrete ramifications - integrate into the globalized economy through the circulation of Chinese junk goods and how this movement contributes to unprecedented rural-urban relations. 
The subject is successively the "Chinese junk trade route", the trading places, the commercial mobilities and finally the network logics. The article concludes by highlighting the decisive role of the private sector in structuring this trade route, whose network logics go beyond the urbanrural continuum.

Key-words : trade route, Chinese junk, cheap trade, mobility, network, urban-rural, Cameroon, Chinese-African

Note : pour préserver l'anonymat, les prénoms mentionnés ne sont pas les véritables prénoms des personnes interrogées.

La pacotille chinoise ${ }^{1}$ a inondé les marchés africains : les ballerines clinquantes, les sandales en plastique à fiabilité incertaine (d'ailleurs appelées «sans-confiances » au Cameroun), les bijoux fantaisie et autres accessoires de mode aussi brillants qu'éphémères, les vêtements de qualité douteuse, les petits appareils électroniques et ustensiles ménagers à vil prix, etc. se retrouvent aussi bien dans les rues marchandes des métropoles que dans les marchés périodiques ruraux. La diffusion massive des produits chinois contribue à faire entrer l'Afrique dans une ère de consommation de masse (Kernen et Khan Mohammad, 2014). La spécificité de ces produits est qu'ils sont largement disponibles, bon marché et adaptés aux faibles revenus des populations urbaines et rurales. En outre, le renouvellement de ces marchandises est important du fait de moindres durabilités et de fortes dépendances à la mode. Les particularités de ces produits favorisent donc leurs circulations d'autant plus que le contexte macroéconomique est favorable.

Les taux élevés de croissance économique en Chine et en Afrique ${ }^{2}$ dans les années 2000 se sont traduits par une multiplication par douze des échanges sino-africains sur la période 2000-2011 ; la Chine est devenue le premier partenaire commercial de l'Afrique en 2009 (Gabas et Chaponnière, 2012). Le Cameroun et la Chine sont des partenaires réguliers depuis le début des années 1970. Les accords bilatéraux de coopération se sont succédés depuis la signature du premier accord commercial en 1972. Si les relations sont anciennes, la période d'accélération de croissance des échanges sino-camerounais a pour point de départ le premier forum sur la coopération sino-africaine tenu à Pékin en 2000 (Cabestan, 2015). Au mois d'avril 2019, ce n'est pas moins qu'un accord sur le financement par la Chine - via un don sans contrepartie pour la construction du nouveau siège de l'Assemblée nationale qui a été signé entre les deux pays. La concrétisation de ce projet hautement symbolique - quand les murs du pouvoir législatif souverain sont payés par une puissance «amie »- datant de plusieurs années est révélatrice de l'ampleur et de la durabilité des liens sino-camerounais. La valeur des importations camerounaises en provenance de la Chine a augmenté de $406 \%$ de 2005 à 2012

\footnotetext{
${ }^{1}$ L'expression « pacotille chinoise » se réfère à la marchandise de qualité inférieure et de peu de valeur fabriquée et importée de Chine - définition départie d'une vision péjorative. On est bien conscients que la gamme des produits fabriqués en Chine comporte aussi des produits de bonne qualité. Or, ce n'est pas cette dernière que l'on retrouve en masse dans les marchés fréquentés par des populations au pouvoir d'achat bas.

${ }^{2}$ D'après la Banque mondiale, de 2000 à 2018, le PIB (en \$ courants) a augmenté de $282 \%$ au Cameroun et de $1023 \%$ en Chine, https://donnees.banquemondiale.org/ (consulté le 26 juillet 2019).
} 
(Cabestan 2015). En 2017, elles représentent un peu plus d'un milliard de dollars alors qu'elles n'étaient que de de 318 millions de dollars en 2012, soit une augmentation de $215 \%$ en cinq ans. La Chine est la première source des importations camerounaises $(18 \%)^{3}$.

Ces valeurs macroéconomiques ne sauraient masquer un taux de pauvreté de $37 \%$ en 2014 et un RNB par habitant (\$PPA internationaux courants) de $3700 \$^{4}$ en 2018 , soit le $153^{\text {ème }}$ rang mondial pour le Cameroun. Des blocages structurels urbains et ruraux persistent, par exemple l'absence d'horizon professionnel viable pour une jeunesse pourtant formée. Dans les campagnes dynamiques de l'Ouest Cameroun, la faiblesse des terres disponibles amoindrit les perspectives d'accumulation via l'agriculture pour une grande partie des jeunes. Le sousemploi, certes en faible recul, est encore estimé à environ $70 \%$ au milieu des années 2010 (INS, 2015). L'univers de la débrouille (Ela 1998) reste l'espace-temps d'une majeure partie de la population qui trouve dans le secteur informel des opportunités entrepreneuriales, fussent-elles marquées par la précarité. L'Institut National de la Statistique au Cameroun estime que la proportion d'actifs engagés dans le secteur informel se situe autour de $90 \%$ dans les années 2010 (INS, 2015). Il existe un paradoxe entre l'importance tangible, quotidienne, la légitimité sociale, la contribution à l'économie nationale du commerce dit « informel » et l'environnement politique ambigu voire conflictuel dans lequel il évolue (Racaud et al., 2018). Par exemple, les vendeurs de rue, dont certains sont le dernier maillon urbain de routes transnationales, sont considérés comme un signe de sous-développement (Bromley, 2000) et comme hors-la-loi dans la plupart des villes des Suds (Rover et Skinner, 2016). Au Cameroun, les déguerpissements réguliers ou exceptionnels de vendeurs de rue font partie de la gouvernance des espaces publics (Yemmafouo, 2018). Quoi qu'il en soit, l'économie informelle est caractérisée par un fort dynamisme économique, une grande flexibilité, une adaptation rapide à la demande (Chen, 2012) et une précarité relative. Le commerce bon marché, notamment du fait des moindres capitaux nécessaires pour y entrer, est une illustration de l'informel, notion essentiellement associée à l'urbain. Les spécificités de la pacotille chinoise, le cadre macroéconomique et les blocages économiques sont des moteurs de l'amplification des circulations de la pacotille chinoise à la ville et à la campagne.

L'entrée par l'objet « route marchande des pacotilles chinoises » oriente l'approche d'une part vers les acteurs du bas, ceux qui construisent par leurs pratiques du commerce bon marché des ramifications discrètes de routes marchandes transnationales, et d'autre part, vers les lieux marchands des circulations. Il est question d'analyser la spatialité de la globalisation, la manière dont des espaces considérés comme périphériques sont parties prenantes de la mondialisation, d'une «mondialisation discrète » (Choplin et Pliez, 2015 ; Pliez 2007). Pour ce faire, l'article interroge comment des marges rurales et des villes secondaires - ramifications discrètes - au Cameroun s'intègrent à l'économie mondialisée via la circulation de la pacotille chinoise et comment ce mouvement contribue à des relations rural-urbain sans précédent ? Le rôle d'une communauté marchande dans les échanges transnationaux a été analysé par Alain Tarrius (2002) mais à l'échelle transméditerranéenne et au sujet de relations économiques relevant pour une bonne part de l'économie souterraine - «mondialisation par le bas ». Par ailleurs, si les

\footnotetext{
${ }^{3}$ Données issues de l'Observatoire of Economic Complexity qui utilise les données de UN Comtrade pour les données à partir de 2012, https://oec.world/en/profile/country/cmr/ (consulté le 26 juillet 2019).

${ }^{4}$ Banque mondiale, le RNB en PPA est le revenu national brut converti en dollars internationaux courants au moyen des taux de parité des pouvoirs d'achat $\underline{\text { https://donnees.banquemondiale.org/indicator/NY.GNP.PCAP.PP.CD?locations=CM }}$ (consulté le 26 juillet 2019).
} 
études sur les circulations de produits chinois en Afrique portent sur les vitrines urbaines dans les centralités commerciales transnationales (Bertoncello et Bredeloup, 2007; Belguidoum et Pliez, 2015 ; Doron 2015), notre démarche combine une géographie rurale, urbaine et des circulations - géographie du commerce bon marché - pour lire les interconnexions d'échelles dans l'espace urbain-rural.

Le matériau empirique provient d'enquêtes de terrain réalisées en $2014^{5}$ et en 2015 . Les données ont été récoltées en suivant la route des pacotilles chinoises, de Douala, la porte d'entrée, aux confins ruraux de l'Ouest Cameroun: six marchés périodiques des monts Bamboutos. La « road research» s'appuie sur une quarantaine d'entretiens semi-directifs avec des commerçants - commerçants sédentaires ou itinérants, importateurs discrets -, un tiers en ville et deux tiers à la campagne ${ }^{6}$. L'approche est inspirée des enquêtes multi-situées (Marcus, 1995) pour mettre en évidence les dynamiques spatiales le long de la route telles les mobilités des marchands, les circulations des objets et l'évolution des centralités commerciales.

Afin de mettre en lumière l'intégration des relations rural-urbain à l'économie mondialisée via la route marchande des pacotilles chinoises, l'article examine dans un premier temps le concept de «route marchande » au regard des relations rural-urbain. Le propos porte ensuite sur les lieux commerçants, puis sur les mobilités et enfin sur l'accès aux réseaux marchands. L'article conclue sur le fait que les acteurs privés jouent un rôle déterminant dans la structuration de cette route marchande dont les logiques de réseau dépassent le continuum urbain-rural.

\section{Les routes marchandes dans le champ des relations urbain-rural afin de lire les interconnexions d'échelles}

On s'intéresse à la section de la route transnationale des pacotilles comprise entre les marges rurales et les villes secondaires de l'Ouest Cameroun. L'analyse des ramifications discrètes qui relient des campagnes et des villes s'inscrit dans les réflexions sur les relations urbain-rural en Afrique. Le regard dual sur ce thème a d'abord insisté sur le rôle négatif de la ville sur les campagnes. Les grandes villes étaient perçues comme un frein au développement rural, comme un centre accaparant les investissements, exploitant la classe pléthorique des paysans et les campagnes dominées, ainsi que l'a théorisé Lipton dans les années 1970. Cette approche dichotomique a été remise en question à partir des années 1980. Un décentrement du regard a permis de mettre en évidence comment la campagne pouvait tenter de subvertir le système en développant par exemple le vivrier marchand (Chaléard, 19967). L'urbanisation était présentée en tant qu'opportunité pour les campagnes les mieux connectées à la ville, elles trouvaient dans le marché urbain un débouché relevant de logiques de marché national ou régional (Chaléard et Dubresson, 1999). Ces travaux appréhendaient la ville et la campagne ensemble. Les interdépendances et complémentarités ont été mises en évidence dans les politiques de développement et de lutte contre la pauvreté (Tacoli 2006, UN-Habitat 2015). Les rapports

\footnotetext{
${ }^{5}$ La campagne de terrain de 2014 a été financée par le LABEX SMS, référence ANR-11-LABX-0066.

${ }^{6}$ Il s'agit de suivre les objets et les personnes dans l'espace et dans une moindre mesure dans le temps (e.g. biographies); le chercheur est directement engagé dans la circulation, le mouvement est le moteur de l'approche. A partir d'un point de départ, on remonte (à partir d'un marché rural) ou on suit (à partir d'un comptoir urbain) la route (objets et marchands). La circulation est alors appréhendée dans diverses situations urbaines ou rurales. Néanmoins, la multiplication des lieux d'enquêtes permet difficilement des enquêtes ethnologiques approfondies sur un lieu.

${ }^{7}$ Voir l'entretien de COM avec Jean-Louis Chaléard dans ce numéro des Cahiers d'Outre-Mer.
} 
ville-campagne étaient surtout examinés à travers les relations agriculture-urbanisation (Tacoli 1998, Pélissier 2000) complexifiées après les libéralisations des agricultures et le mouvement de diversification des revenus ruraux - désagrarianisation - (Bryceson, 1999). Des études sur des économies extractives, l'industrie ou l'artisanat ont pris en compte la globalisation dans les relations urbain-rural (Agergaard et al., 2009) mais le petit commerce, pourtant attrayant pour les nombreuses populations à faible capitaux reste une périphérie scientifique.

La «pluriactivité multispatiale »(Lesourd, 1997) marque les sociétés rurales qui jouent de plus en plus de la complémentarité ville-campagne ou des diversités d'espaces à plusieurs échelles (Charlery de la Masselière, 2013). Le développement du commerce bon marché de produits importés de Chine s'inscrit dans ces logiques combinatoires et rend compte d'interconnexions inédites du monde rural à d'autres échelles. Les connexions de paysanneries (notamment montagnardes) avec des réseaux de long cours ne sont toutefois pas nouvelles puisque les filières des cultures d'exportations coloniales (e.g. café, thé) ont introduit les producteurs dans le marché mondial. Néanmoins, c'est le système colonial puis post-colonial, à travers l'Etat, qui en était le moteur et les paysans ne décidaient de pas grand-chose pour ces cultures (e.g. au sujet des prix, des normes) alors que l'essor du commerce bon marché repose sur des acteurs du bas tels des paysans commerçants, des marchands itinérants ruraux, des vendeurs urbains et des entrepreneurs transnationaux. Ces acteurs du quotidien «font avec» l'environnement socio-économique pour en extraire des opportunités à moindre coût en connectant le local à d'autres échelles.

Les dynamiques locales prennent leur sens dans un ensemble plus vaste, les formes géographiques de la route des pacotilles dans l'Ouest du Cameroun sont à comprendre dans leurs relations avec d'autres échelles. Massey (1991) a proposé l'expression « a global sense of place» pour qualifier l'enchâssement entre le global et le local, indispensable à la compréhension d'un lieu. Notre point de départ est la circulation urbain-rural pour observer les interconnexions d'échelles puisque nous cherchons à comprendre les modalités d'intégration de marges rurales, de villes secondaires et des relations urbain-rural à l'économie mondialisée. Les routes globales sont celles de l'échange marchand qui intègrent les dynamiques de la mondialisation et également les opportunités et contraintes locales (Pliez, 2007). Ce choix d'une géographie des circulations urbain-rural ouvertes combine des apports de géographie rurale et de géographie urbaine afin de mettre en évidence les relations entre les objets « urbain » et « rural » étant donné qu'on s'accorde à penser que les relations sont constitutives des objets mêmes. Jean-Louis Chaléard et Alain Dubresson (1999), à travers une géographie des relations, avaient pris le parti du dépassement d'approches cloisonnées sur ces objets en proposant des analyses sur ce qui unit et désunit villes et campagnes. La route marchande articule ces objets ainsi que leurs relations à d'autres échelles.

La route marchande combine les dimensions spatiales de la circulation avec des aspects relationnels entre acteurs. Dans sa conception spatiale, la route se réfère à l'ensemble de lieux, reliés de manière continue ou discontinue. Dans ce dernier cas, la figure de la route est une métaphore. Cependant, la route est aussi l'infrastructure de transport, la route matérielle, les dispositifs logistiques. La route marchande matérielle renvoie aux grands investissements et aux accords bilatéraux (en particulier avec la Chine). Plus discrètement, la route parvient aux confins des montagnes, dans ce cas elle prend souvent la forme d'une piste boueuse ou caillouteuse suivant la saison. La route des pacotilles, c'est aussi l'ensemble des acteurs reliés entre eux par la commercialisation, c'est une conception immatérielle qui permet l'articulation 
de réseaux sociaux et de réseaux spatiaux (Belguidoum et Pliez, 2012). Elle est en partie inspirée d'éléments de la Global Commodity Chain (Gereffi et Korzeniewicz, 1994) complétée ensuite par la Global Value Chain (Gereffi et al., 2005) qui prend mieux en compte les aspects institutionnels (normes, régulation, etc.). Ces approches économiques induisent une rigueur méthodologique difficilement applicable pour des objets imbriquant informel et formel, et dont la régulation est flexible voire ambigüe (Choplin et Pliez, 2015). Choplin et Pliez (2015) soulignent que le concept de « route » est plus souple et rend mieux compte de la dimension réticulaire du commerce. Elle permet d'étudier les dynamiques peu visibles ou en relative marge des Etats, les processus discrets d'interconnexion d'échelles « to understand the ways in which networks of people trace out paths which, by creating new destinations, by the emergence of forms of organization based on trust and reputation, and by establishing unexpected regulations combining public and private action, strengthen the links between the places involved and locate these places within global trade routes » (Choplin et Pliez, 2015 : 6). La mise en exergue des assemblages entre réseaux spatiaux et réseaux sociaux par la route commerciale est par ailleurs une entrée pour interroger d'une part les « dislocations et recompositions spatiales en Afrique subsahariennes induites ou amplifiées par la crise des complexes politico-économiques ou par [ ] la mondialisation/globalisation » (Dubresson et Raison, 2002 : 119) et d'autre part la « discordance des temps et l'interférence des espaces » de mondes ruraux ouverts (Charlery de la Masselière, 2014).

Au total, centrer le regard sur les ramifications discrètes de la mondialisation dans la région Ouest du Cameroun revient à porter l'attention sur comment cette région est partie prenante de la mondialisation. Cela informe d'un côté sur les processus locaux et d'un autre côté sur les phénomènes globaux. Des principes de la totalité se retrouvent dans les parties, il est donc possible d'enrichir la connaissance de l'un ou de l'autre par l'un et l'autre, ce qu'Edgar Morin nomme le principe hologrammatique (Morin, 1977). En ce sens, la partie - ramification discrète - illustre le tout - route globale -, sans le résumer, et le tout informe sur la partie. Que disent les lieux marchands dans l'Ouest camerounais de la route transnationale des pacotilles importées de Chine?

\section{Marchés urbains et marchés ruraux : centralités commerciales complémentaires}

L'Ouest du Cameroun est une région agricole qui alimente les marchés urbains camerounais et sous-régionaux depuis l'essor du vivrier marchand. Les filières café dans un premier temps, puis la route du vivrier marchand a intégré le pays Bamiléké - groupe autochtone et principal dans la région - au marché. Le marché central de Bafoussam (marché A) et les marchés périodiques ruraux des monts Bamboutos sont des carrefours entre les routes marchandes, des points d'entrée ou de sortie, des interfaces entre plusieurs échelles, et donc des lieux privilégiés d'analyse.

\section{Le marché A à Bafoussam : centre régional de la pacotille chinoise}

Située à $270 \mathrm{~km}$ au nord-est de Douala, Bafoussam est la capitale régionale de l'Ouest. Elle serait la troisième ville du Cameroun (Yemmafouo, 2018), sa population croît rapidement, elle pourrait atteindre 420000 habitants en $2019^{8}$. L'attractivité de Bafoussam repose en particulier

\footnotetext{
${ }^{8}$ Le dernier recensement de 2005 indique une population de 239287 habitants. L'estimation pour l'année 2019 s'appuie sur le taux de croissance annuel moyen de 4,1\% sur la période 1987-2005 publié par le Bureau Central
} 
sur ses fonctions de chef-lieu et sur ses fonctions commerciales (Yemmafouo, 2013). La ville bénéficie d'une position stratégique puisqu'elle est au carrefour des axes reliant la région NordOuest avec Douala et Yaoundé. Située dans une région agricole primordiale, Bafoussam joue le rôle de centre de collecte et de redistribution des productions de son arrière-pays que l'on retrouve dans les marchés urbains camerounais et sous-régionaux. Bafoussam est aussi le comptoir régional des marchandises importées de Chine. Cette centralité commerciale assure donc des fonctions logistiques pour ces deux routes marchandes. D'un côté elle oriente des flux de l'arrière-pays vers le marché sous-régional, des flux sortants. D'un autre côté, elle organise des flux entrants, en provenance de Chine, via le port de Douala.

Les deux principaux marchés intra-urbain où l'on trouve des produits manufacturées sont par ordre décroissant le marché A et le marché $\mathrm{B}^{9}$. Construit en 1972 pour décongestionner le marché $\mathrm{A}$, le marché $\mathrm{B}$ est équipé de 1273 boutiques et de 175 étals sur une superficie de 1,5 ha (Nokam, 2012). Il assure essentiellement du commerce de détail contrairement au marché A, nœud régional du commerce. Fondé en 1924, il est situé au cœur de la métropole régionale, au carrefour de deux routes nationales. Le marché A assure une partie de l'approvisionnement des marchés de Dschang et Mbouda, respectivement chef-lieu du département de la Menoua et de celui des Bamboutos. Ces villes compteraient environ 150000 habitants et sont équipées d'un marché central qui assure du commerce de détail. Les commerçants se ravitaillent auprès de grossistes à Bafoussam ou à Douala s'ils disposent de capitaux importants. Leur clientèle est essentiellement composée de consommateurs urbains mais de petits revendeurs peuvent y faire des achats. Ces deux marchés jouent un rôle secondaire dans la structuration de la route marchande contrairement au marché A.

La superficie du marché A serait d'après les autorités municipales de 3,5 ha (Ibidem) mais l'espace marchand a débordé de l'implantation initiale. Une myriade de boutiques et de kiosks se sont accumulés hors de l'enceinte initiale, remplaçant les habitations ou remplissant la rue. La limite du marché est confuse.

des Recensements et des Etudes de la Population en 2010. BUCREP, 2010. Rapport de présentation des résultats définitifs 2005. Yaoundé, Bureau Central des Recensements et des Etudes de Population, 67 p.

${ }^{9}$ En périphérie, de la ville, le marché Casablanca est un marché de gros ouvert pour les vivres. Il est un lieu de collecte et de redistribution qui organise des flux sortants, ceux des denrées venant de l'arrière-pays. 


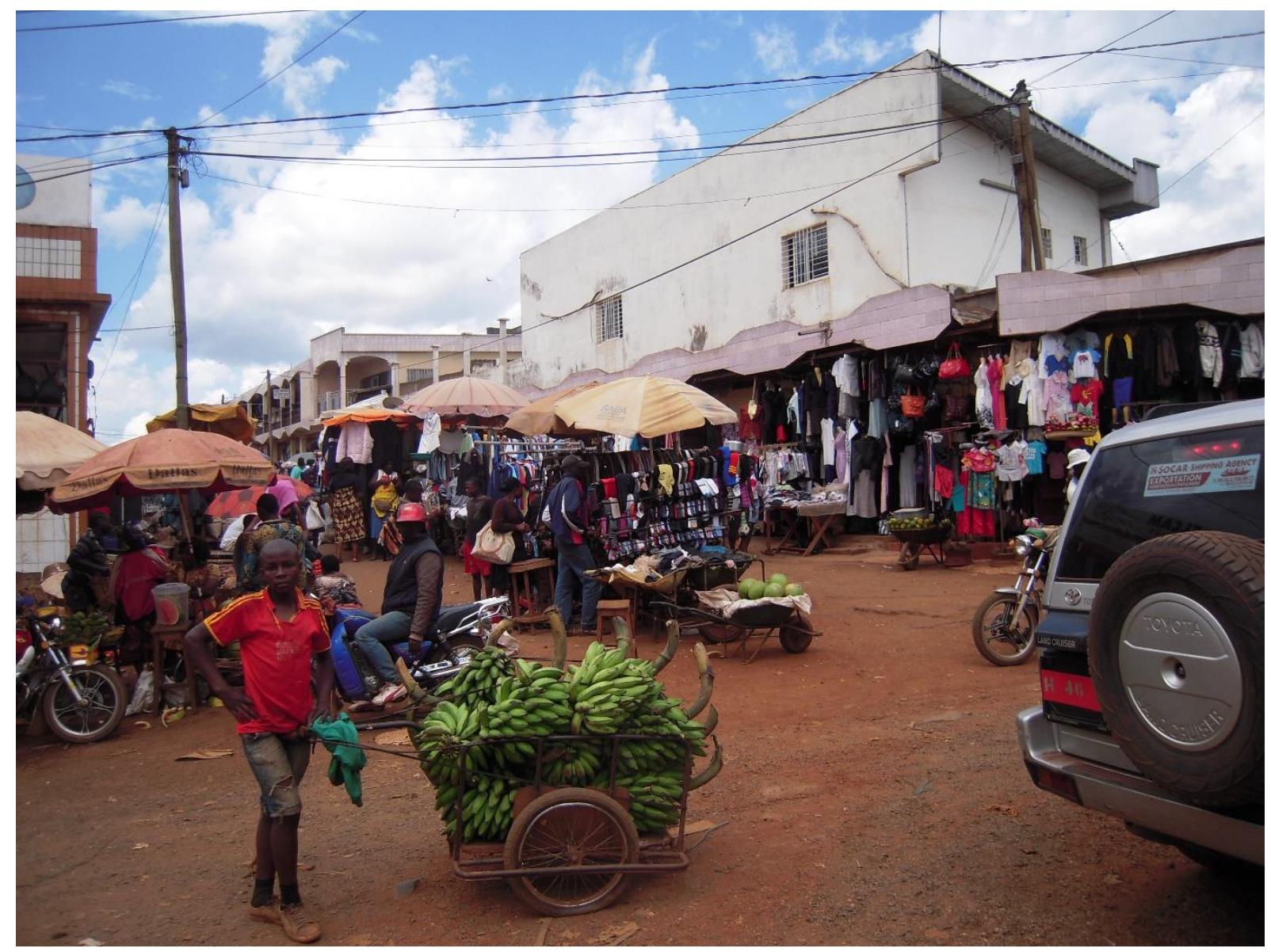

Fig. 1. Limites confuses du marché A, Bafoussam (Racaud, 2014). Légende : l'espace commercial a débordé de l'implantation initiale.

Le marché est officiellement constitué de 2380 boutiques et de 534 étals, soit 2914 commerçants (Ibidem). Si on y trouve des commerces de denrées agricoles, les échanges principaux concernent les produits manufacturés. Cette pierre angulaire du commerce est composée d'innombrables dispositifs variés de vente, de la boutique aux kiosks mobiles, agencés dans un dédale d'artères le plus souvent étroites. A première vue, ce lieu marchand congestionné pourrait sembler désordonné, or il n'en est évidemment rien.

Le marché est divisé en quatre secteurs. Le secteur 2 et le secteur 3 sont composés d'étals sur lesquels sont essentiellement disposés des denrées. Le secteur 1, au cœur du marché originel, et le secteur 4 sont les hauts-lieux de la marchandise importée de Chine. Les articles sont vendus dans des boutiques, kiosks ou mini-entrepôts dont la taille n'excède pas $25 \mathrm{~m}^{2}$. La marchandise s'étale aussi dans les allées et passages discrets. Elle est stockée dans des cartons, des sacs, disposés sur des présentoirs, des étagères, superposés dans des enchevêtrements de cintres suspendus, etc. L'identité des différentes sections repose sur les types de produit, elle se retrouve dans la toponymie explicite des sections. Par exemple Dubaï Center est spécialisé dans le petit électronique, les accessoires de modes (bijoux fantaisie, maquillage, etc.) alors que Secteur chinois est spécialisé dans les vêtements, les ballerines, les sacs à main et les sandales. De plus, dans ces sections, on observe des spécialisations plus fines. Le secteur chinois comporte une zone pour les sandales basiques et meilleur marché, distincte de la zone des ballerines, des chaussures à talon, des baskets et des sacs à main. Le produit caractérise l'espace 
marchand, la spécialisation jouant un effet significatif sur l'identification et l'attractivité des sous-secteurs.

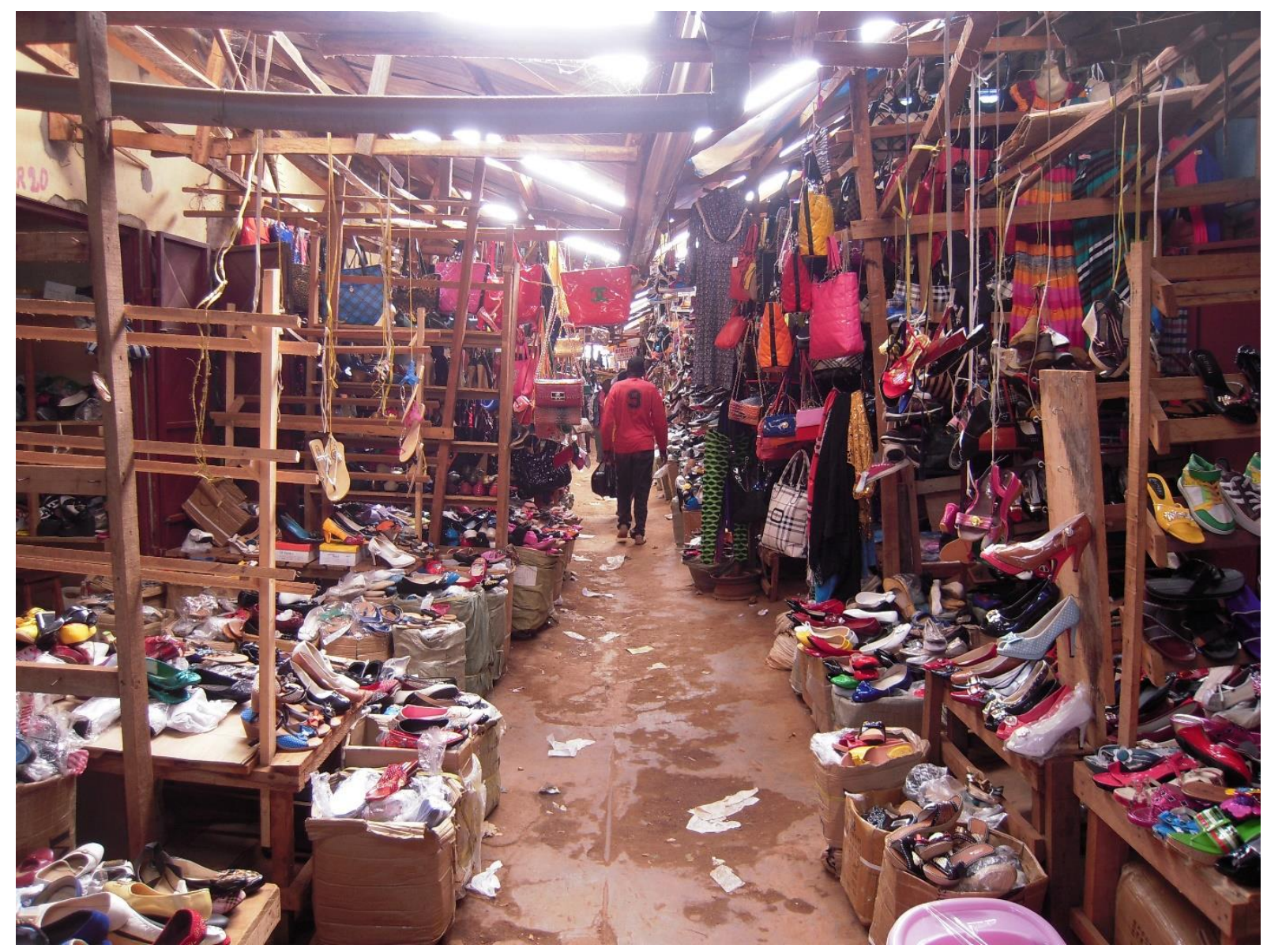

Fig. 2. Section des chaussures sophistiquées et des sacs à main dans Secteur chinois, marché A, Bafoussam (Racaud, 2014) 


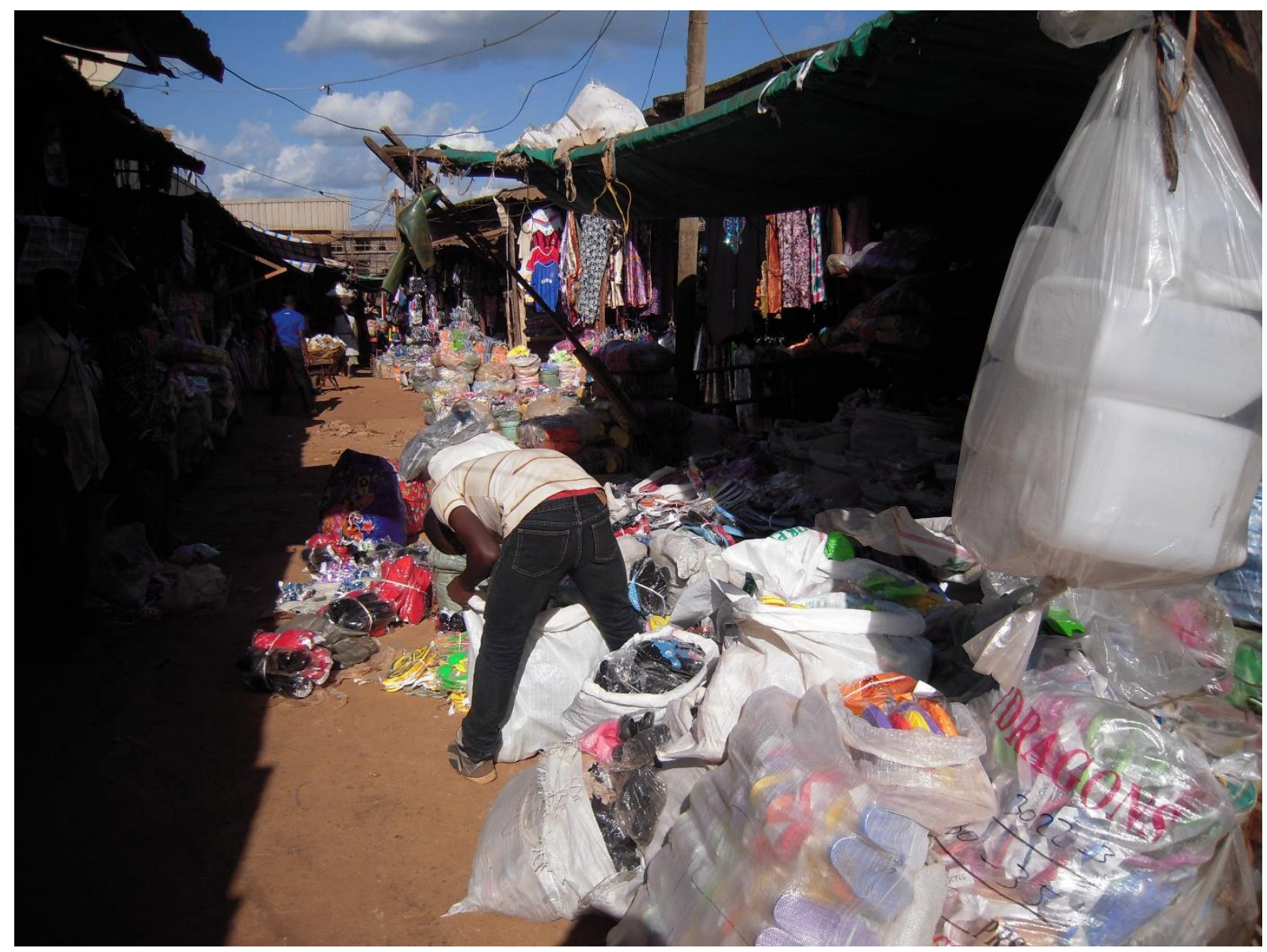

Fig. 3. Section des sandales basiques dans Secteur chinois, marché A, Bafoussam (Racaud, 2014)

Les figures 2 et 3 montrent une grande quantité d'articles mais on ne voit pas de clientèle abondante. Les entretiens révèlent que la compétition s'est beaucoup intensifiée à partir des années 2010, la période faste semble derrière. D'une manière générale, les commerçants, qu'ils vendent dans un kiosk ou dans une boutique s'accordent à dire que le marché est saturé «c'est maintenant mort, ça ralentit, ça ralentit et ça ne fait que ralentir » (Diane, vendeuse de sandales). L'augmentation du nombre de commerçants est un indicateur de la concurrence. Des grossistes en vêtements made in China déclarent que leur nombre aurait été d'une quinzaine au début des années 2010 alors qu'ils seraient une quarantaine cinq ans plus tard au marché A. La concurrence s'observe également par la difficulté à accéder à la jouissance d'un point de vente. A ce propos, Nokam écrit «A Bafoussam, obtenir une place sur le marché relève d'un véritable parcours du combattant $\gg(2012$ : 98). L'accès est possible via trois moyens : la sous-location illégale - à un bailleur, l'achat individuel, et la subdivision d'un point de vente entre plusieurs vendeurs (Nokam, 2012). Etant donné la forte demande en espaces marchands, les prix des transactions foncières (sous-location, transfert de bail) auraient augmenté. Le montant des loyers des boutiques communales est pourtant, en principe, encadré par une délibération du Conseil de la Communauté urbaine de Bafoussam (CUB). Or, certains bailleurs parviendraient à accumuler des boutiques en vue de leur sous-location. Ce marché foncier illégal a fait l'objet de scandales atteignant les plus hautes autorités régionales. En 2011, Gabriel Mboucheka, chef du service de l'assiette fiscale, proche collaborateur d'Emmanuel Nzete, Délégué du gouvernement à la tête de la Communauté urbaine de Bafoussam, est impliqué dans une escroquerie au marché A. En 2018, le prévenu est finalement condamné à sept ans de prison 
pour avoir reçu de l'argent de commerçants contre des promesses d'attribution de nouvelles boutiques au marché A. En 2019, cette affaire est toujours sujet à controverses entre Kayo Kengni, mandataire du collectif des commerçants du marché A et le Délégué du gouvernement auprès de la CUB. Le premier, accuse le second, via les réseaux sociaux et la presse, de l'avoir floué de même que d'autres commerçants pour l'attribution de nouvelles boutiques. Cette affaire met en lumière l'opacité quant à la gestion et l'attribution des boutiques du marché $\mathrm{A}$. Les projets de construction et d'attribution de nouvelles boutiques ne font qu'éclairer cette nébuleuse et ne sont d'ailleurs pas l'apanage du Marché A puisque des dynamiques proches ont été analysées en Afrique du Sud (Pezzano, 2016) ou au Kenya (Racaud, 2018). La réponse de février 2019 que le Délégué du gouvernement adresse au mandataire du collectif des commerçants illustre assez bien le potentiel d'institutionnalisation du clientélisme « Le collectif [des commerçants] peut par ailleurs solliciter respectueusement à Monsieur le Délégué du Gouvernement des opportunités d'attribution de boutique dans les nouveaux espaces marchands et Monsieur le Délégué du Gouvernement pourra apprécier la faisabilité de cette sollicitation en sa qualité de bon père de famille ${ }^{10}$. Cela révèle également les imbrications entre les sphères politiques et économiques dans la gouvernance d'un marché, objet de convoitise.

La gouvernance urbaine est empreinte de rivalités de pouvoir entre, schématiquement, la CUB représentée par le Délégué du gouvernement, lui-même nommé par le pouvoir central, et les maires élus des trois communes urbaines d'arrondissement ${ }^{11}$. La CUB est une création du pouvoir central en 2008 pour notamment contrebalancer le poids de l'opposition politique. Bafoussam est un bastion traditionnel du SDF (Social Democratic Front), c'est-à-dire le parti historique d'opposition au RDPC (Rassemblement du Peuple Camerounais), parti du Président Paul Biya et parti de gouvernement depuis $1984^{12}$. Pourtant, lors des élections municipales de 2013, le RDPC parvient à arracher au SDF la mairie de Bafoussam $1^{\text {er }}$ et celle de Bafoussam $3^{\text {ème }}$. Le SDF pèse toujours dans ces conseils municipaux où les prises de décisions sont freinées par les clivages partisans. Le morcellement administratif est ainsi tâché d'antagonismes politiques : «la difficile cohabitation entre les maires d'arrondissement et l'autorité centrale entrave la gestion équitable des ressources financières. Les querelles et les conflits de compétences entre les instances sont responsables de la décadence de la ville et de ses infrastructures »(Nokam, 2012: 110). La fragmentation administrative se double d'une concentration du pouvoir dans la CUB.

Par ailleurs, les représentants des commerçants ont de nombreuses revendications à l'endroit de la CUB. L'association des commerçants des marchés de Bafoussam (ACOMAB) est le principal porte-parole du secteur privé. Les marchands s'élèvent en particulier contre

\footnotetext{
${ }^{10}$ Camer.be, mercredi 6 février 2019, «Collectif des commerçants: Emmanuel Nzété réfute tout lien de parenté avec Mboucheka Gabriel », https://www.camer.be/72969/11:1/cameroun-collectif-des-commercants-emmanuelnzete-refute-tout-lien-de-parente-avec-mboucheka-gabriel-cameroon.html (Consulté le 6 août 2019).

${ }^{11}$ Depuis 2008, Bafoussam est une Communauté Urbaine avec à sa tête un Délégué du gouvernement. Il exerce son autorité sur les trois communes d'arrondissement (Bafoussam $1^{\text {er }}$, Bafoussam $2^{\text {ème }}$ et Bafoussam $3^{\text {ème }}$.) dont les maires sont élus aux élections municipales. Les dernières élections municipales se sont tenues en 2013, les suivantes devaient se tenir en 2018 mais elles ont été reportées à deux reprises. En juillet 2019, le Président Biya annonce que le mandat des conseillers municipaux élus lors du scrutin du 30 septembre 2013 est prorogé jusqu'au 29 février 2020.

${ }^{12}$ Le RDPC est une refonte en 1984 de l'UNC (Union Nationale Camerounaise), parti fondé par le Président du Cameroun, Ahmadou Ahidjo en 1960, année de la proclamation de l'indépendance. Le pouvoir central au Cameroun n'a jamais connu d'alternance.
} 
l'augmentation non concertée des prix des loyers, contre des mesures répressives à l'endroit des mauvais payeurs de loyer, ils soulignent le manque de soutien, la persistance d'équipements vétustes et défaillants et last but not least : ils s'insurgent contre l'obligation du paiement de la taxe foncière par les commerçants locataires qui sous-louent - pratique interdite - des boutiques à des bailleurs. Ce dernier argument montre d'une part que les revendications savent s'accommoder d'une gouvernance ambigüe et d'autre part que les autorités ne rechignent pas à percevoir des loyers de locataires illégitimes. La gouvernance des espaces publics appropriés par les vendeurs de rue à Bafoussam est marquée par un partage du pouvoir relativement négocié mais précaire, par la coexistence instable mais relativement pacifique entre les autorités et les marchands dit «informels» (Yemmafouo, 2018). Cependant, les conflits dans la gouvernance du marché A peuvent prendre la forme d'évacuation forcée « déguerpissements» - parfois d'un grand nombre de vendeurs considérés comme « informels ». En 2012, ce n'est pas moins d'un millier de vendeurs qui a été chassé du marché A, soit environ un tiers de l'effectif officiel de commerçants au marché. On peut donc raisonnablement penser que le nombre réel de commerçants est très éloigné du nombre officiel, et qu'il y en a donc bien plus que 2914. L'intégration du marché A à la route transnationale se réalise via une imbrication entre les économies dite formelles et informelles.

Le marché A est relié à plusieurs comptoirs internationaux. Les grossistes de Bafoussam sont clients d'importateurs installés à Douala : Chinois et Camerounais dans une moindre mesure. Néanmoins, certains importateurs camerounais discrets sont installés à l'intérieur du marché A. On les trouve difficilement car ils sont situés dans une étroite allée excentrée, dont l'entrée est assez invisible. Quelques dizaines de commerçants sont assis dans de minuscules boutiques qui relèvent d'ailleurs plutôt du placard ${ }^{13}$. Ils vendent pour l'essentiel des vêtements, la gamme sportive est la plus courante. Ces «boutiques» sont plutôt des vitrines de la gamme des produits. Le stock n'est pas au marché, il est caché dans des résidences entourées de hauts murs d'enceinte du quartier huppé de Kamkop. Ces commerçants sont bien plus importants qu'ils en ont l'air puisqu'on apprend - laborieusement - qu'ils dédouanent régulièrement des containers au port de Douala. Ces importateurs discrets visent à se soustraire aux taxes, ils ne paieraient pas la patente mais seulement une part de l'impôt libératoire. On imagine qu'étant donné la superficie de chaque « placard», le loyer est modique. Qu'est-ce à dire ? Ces acteurs de l'ombre arriment directement la métropole de l'Ouest du Cameroun aux centralités commerciales internationales telles que Guangzhou, Dubaï ou Bangkok, via des circuits qui cherchent à échapper aux « radars ». Les importateurs de coulisses tirent profit de la globalisation et ils rapprochent finalement les comptoirs internationaux des consommateurs de l'Ouest. Bafoussam est bien plus qu'un simple relais, elle assure, à travers ses connexions invisibles, des fonctions de nœuds dans un réseau international, comme cela a pu être observé dans d'autres villes secondaires en Afrique de l'Est (Mainet et Racaud, 2015). En tant que nœud régional du réseau marchand de la pacotille, Bafoussam est aussi relié à son arrière-pays rural.

\section{Marché ruraux : confins commerciaux de la route transnationale}

Les marchés ruraux jouent le rôle d'interface entre d'une part leur arrière-pays agricole et les marchés urbains, et d'autre part entre les comptoirs commerciaux et les consommateurs des campagnes. Ce sont des hauts-lieux de l'échange, marchand et non-marchand car les marchés

\footnotetext{
${ }^{13}$ Rien n'indique que ce groupe d'importateurs soit le seul au marché A.
} 
périodiques sont de grands moments de la vie rurale (Chaléard, 1996). Les paysans représentent la grande majorité des clients, ils peuvent aussi y vendre des fruits de leur labeur. Ces marchés sont donc attractifs pour les commerçants venus s'approvisionner en denrées à destination des marchés urbains, pour les paysans venus écouler une partie de leur culture, et également pour les commerçants de produits manufacturés. Dans des économies rurales où l'agriculture prévaut, la vitalité d'un marché repose sur celle de l'agriculture. On comprend que l'essor de la route des pacotilles repose dans ces confins ruraux sur le dynamisme de l'agriculture locale.

La densité des marchés périodiques est importante, elle est fonction des densités de population. Ces dernières sont de l'ordre de 200 à 800 hab. $/ \mathrm{km}^{2}$ dans l'Ouest (Yemmafouo, 2013). On dénombre 31 marchés périodiques au pays Yemba accessibles via des pistes pour l'essentiel d'entre eux. (fig. 4). Le pays Yémba est un sous-ensemble du pays Bamiléké situé sur les hautsplateaux de l'ouest du Cameroun. Le pays Yémba correspond à peu près au département de la Menoua, il s'étend de Dschang à l'ouest jusqu'à presque Bafoussam à l'est et Mbouda au nord. Selon la culture Yémba, la semaine dure huit jours, par conséquent, il y a un roulement des jours de marché pour caler à la semaine de sept jours. Le plus souvent, dans un village, le jour de marché se tient une fois tous les huit jours. Le calendrier des marchés en Pays Yémba est un document disponible dans des boutiques en ville nécessaire pour connaître la répartition dans le territoire des jours de marché.

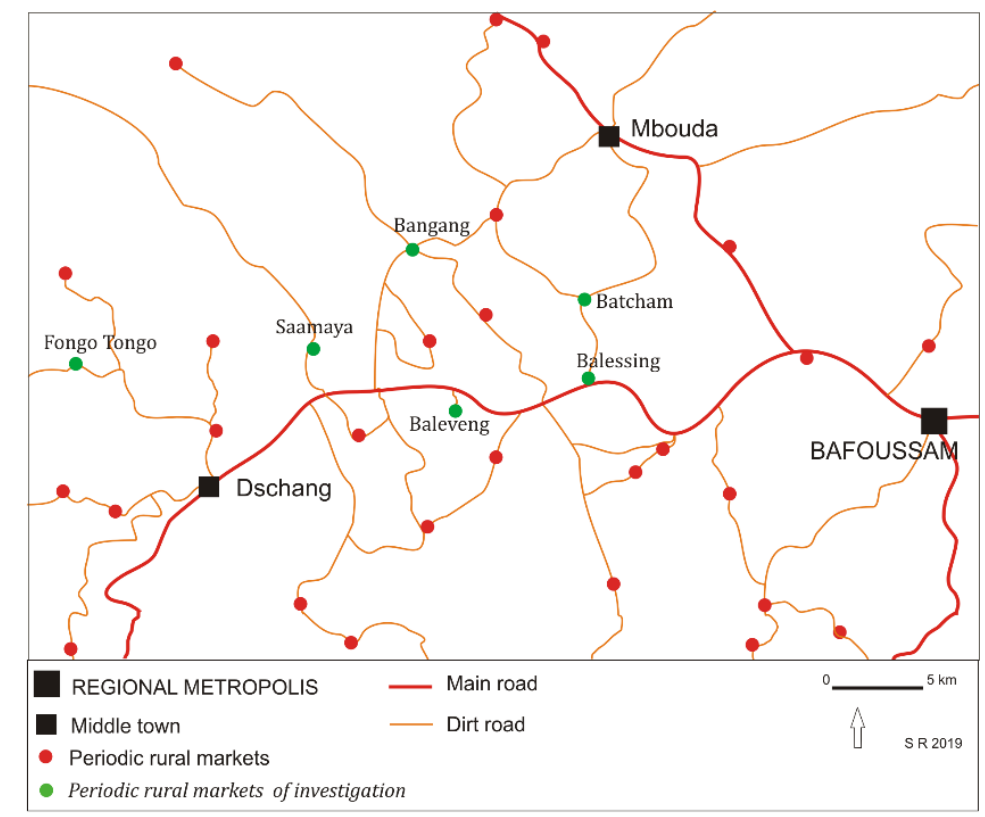

Fig. 4 Carte des marchés en Pays Yémba (Racaud, à partir du calendrier des marchés en Pays Yémba et de Google map)

Les paysages de ces lieux marchands sont marqués par un assemblage de produits à l'image du terroir et d'articles reflétant la consommation de masse. Le marché est, grosso modo, divisé en deux ensembles, celui des denrées agricoles et celui des produits manufacturés, eux-mêmes subdivisés en plusieurs secteurs selon les types de produits. Il y a autant de configurations spatiales que de marchés. Certains sont installés dans des espaces ouverts, avec donc une concentration dans l'espace, d'autres sont incorporés dans les rues des villages selon des dispositifs longilignes (fig. 5 et fig. 6). Globalement, les dispositifs de vente sont sommaires, ils consistent en des tables en bambou, couvertes ou non, d'une taille comprise entre un et six mètres carrés, équipées ou pas de présentoirs verticaux. 


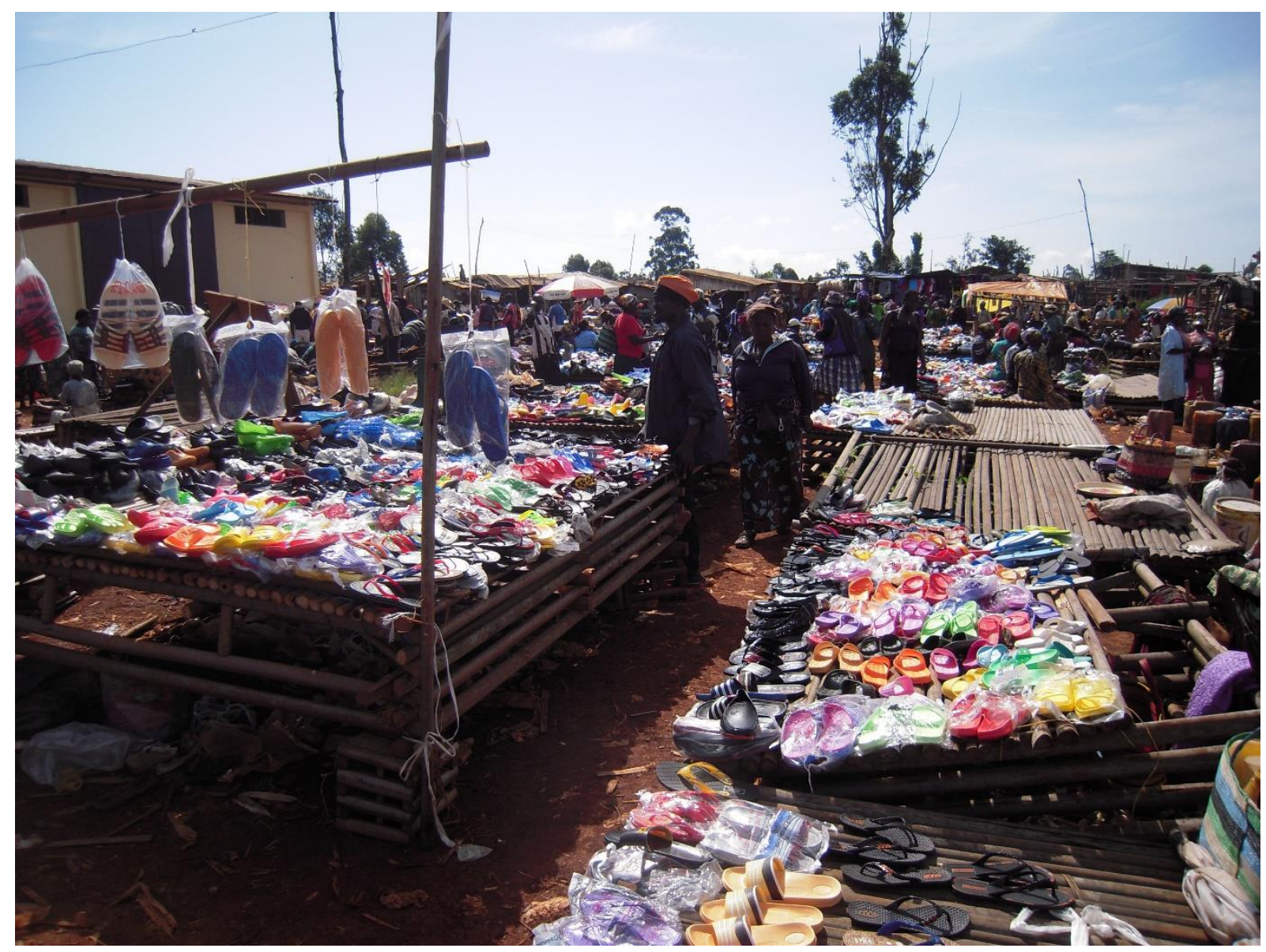

Fig. 5 Secteur des sandales en plastique au marché de Baleveng (Racaud, 2014) 


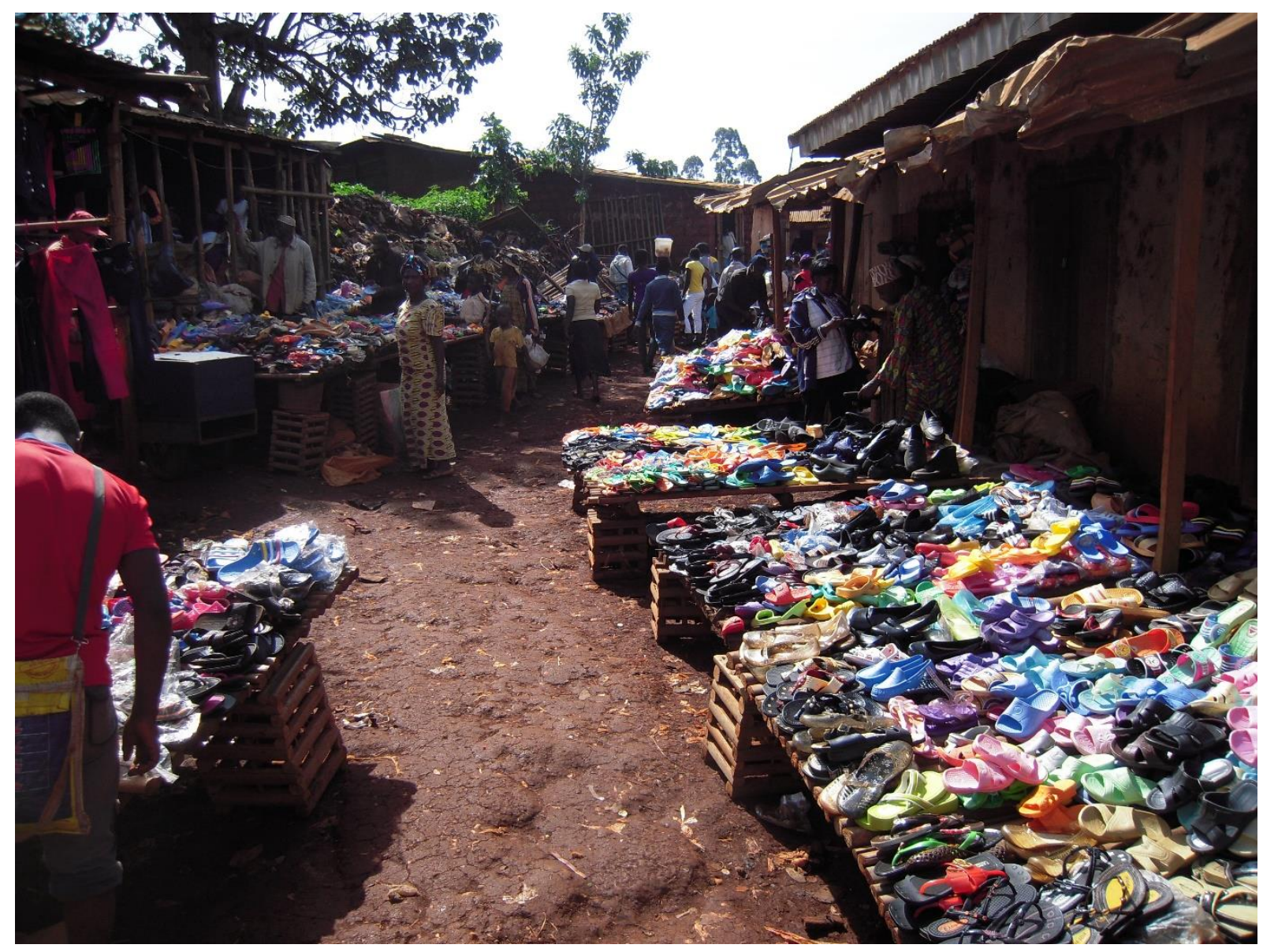

Fig. 6 Secteur des sandales en plastique à Batcham (Racaud, 2014)

La proportion des marchandises importées entre les différents marchés est relativement homogène. Les classements des dispositifs de vente par produits sont relativement similaires : la fripe est le premier groupe de produits, suivi par les vêtements neufs et la layette, puis par la quincaillerie/épicerie ou les sandales/ chaussures en plastique, et enfin par les accessoires de mode.

Fig. 7 Nombre de dispositifs de vente de produits manufacturés dans trois marchés

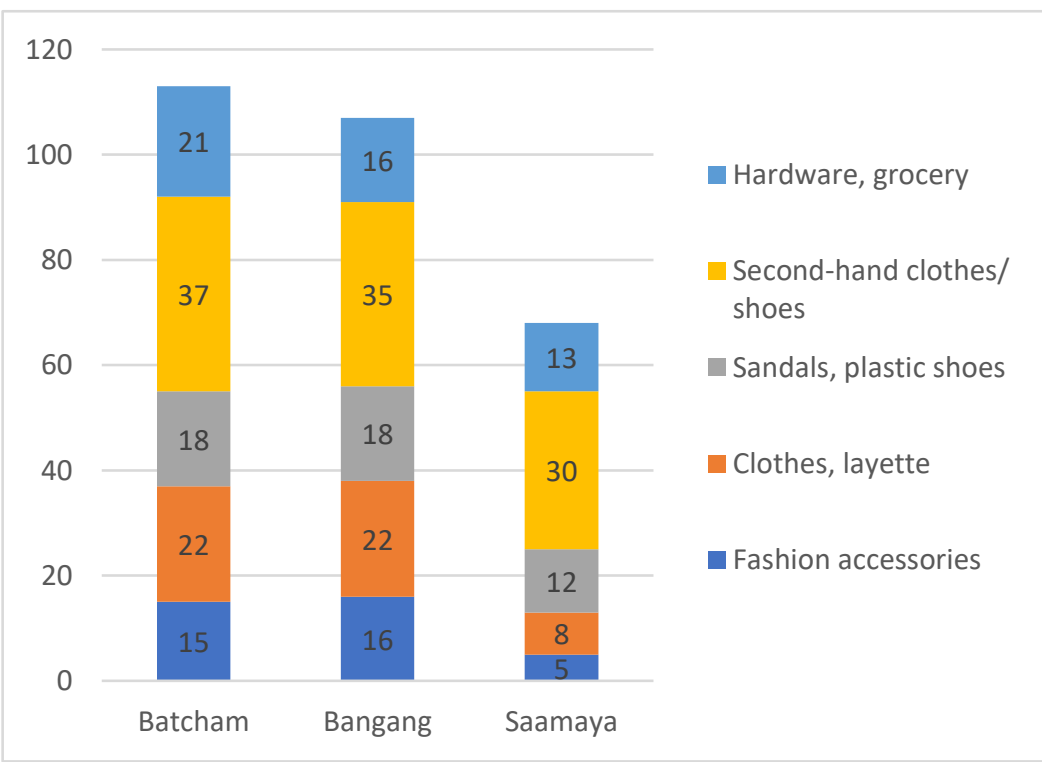


Ces répartitions informent sur le pouvoir d'achat, les habitudes de consommation, la clientèle, voire la démographie. La fripe est meilleur marché, elle correspond à un pouvoir d'achat limité. Le poids du secteur de la layette illustre probablement le nombre élevé d'enfants par femme, il est de 4,7 en 2016 d'après la Banque mondiale, la démographie crée un marché. Avec la diffusion des vêtements importés de Chine, l'habillement est plus abordable, ces articles bénéficient de représentations positives de la part des clientes comme le déclare Christiane rencontrée au marché de Bangang, mère et vendeuse : «les produits chinois ça nous change beaucoup la pauvreté, il n'y en a plus, ceux qui étaient trop sales ne sont plus trop sales, ça nous aide beaucoup ».

Les accessoires de mode ont surtout une clientèle jeune, la jeunesse représente $35 \%$ de la population $^{14}$. Le poids des accessoires de mode indique qu'une part du pouvoir d'achat des ruraux est destinée à des dépenses qui ne relèvent pas de «la survie », ces campagnes dynamiques et jeunes sont entrées dans l'ère de consommation de masse. La distribution de ces articles bon marché importés participe à l'émergence d'une nouvelle culture matérielle (Kernen et Khan Mohammad, 2014). Les jeunes jouent un rôle déterminant puisqu'ils sont les plus investis dans le secteur attractif de la mode. La figure 8 montre les dispositifs de vente de deux jeunes marchands (à gauche avec un polo bleu marine, à droite avec un bonnet couleur rasta) au marché de Batcham. Leur présentoir respectif contient des bijoux en plastique, des sacs à main, du maquillage, des casquettes américaines, des bonnets à l'effigie du Barça, etc. en somme la panoplie d'objets globalisés. La cliente est une jeune fille dont le style n'a rien à envier aux jeunes urbaines. La route de la pacotille contribue au rapprochement entre campagne et ville et à l'intégration du monde rural au monde globalisé.

\footnotetext{
${ }^{14}$ Pourcentage des jeunes âgés de 15-34 ans. Les moins de 25 ans représentent $64 \%$ de la population, l'âge médian est de 17,7 ans et l'âge moyen est de 22,1 ans. D'après l'Institut national de statistique au Cameroun, données produites en 2010 d'après le recensement de 2005.
} 


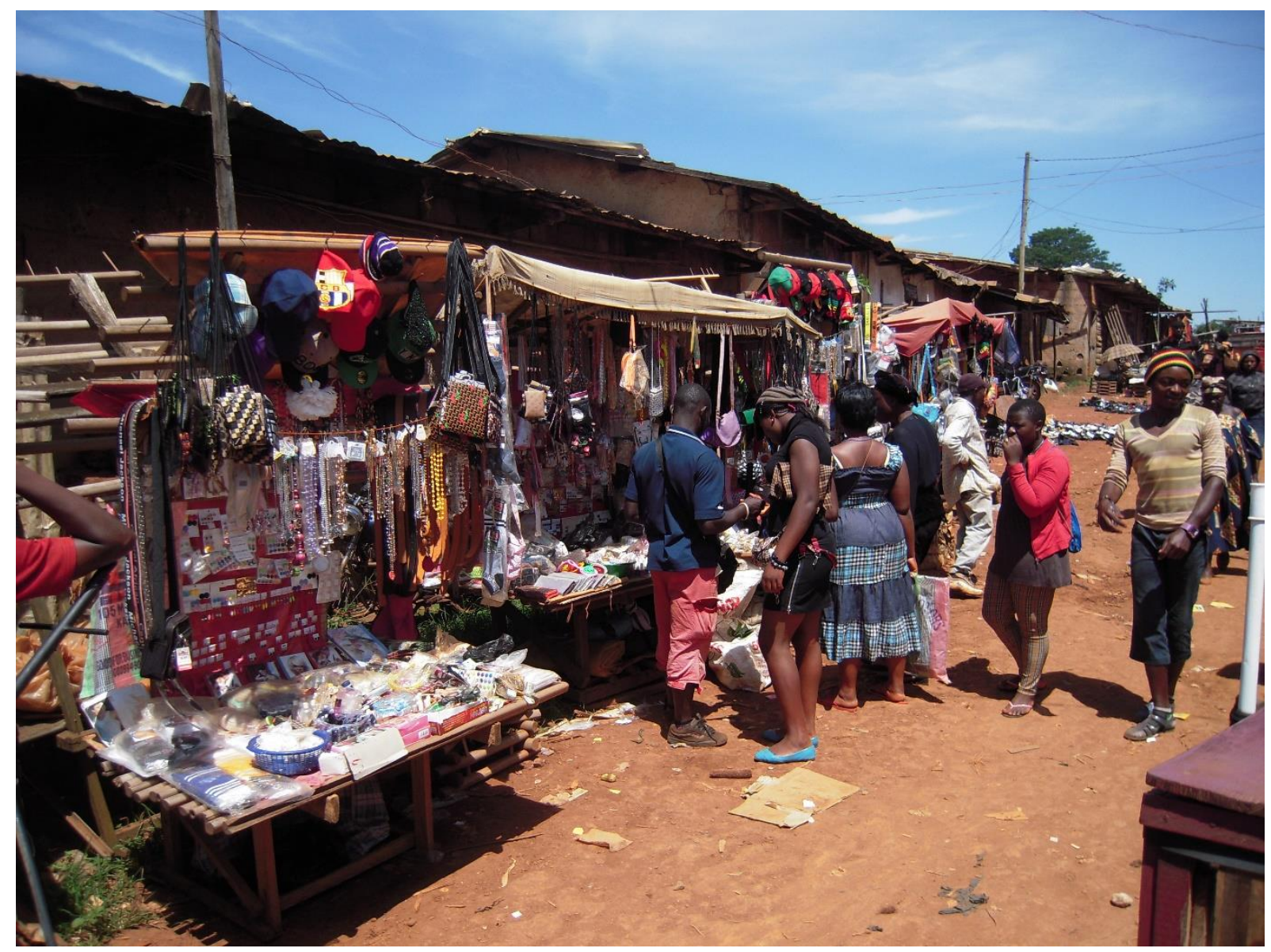

Fig. 8 Jeunesse et objets globalisés dans les campagnes de l'Ouest (Racaud, 2014)

Le marché A et les marchés ruraux organisent à des niveaux différents la circulation de la pacotille chinoise dans ces périphéries de la mondialisation. Cette dernière s'appuie donc sur des lieux ancrés d'une part dans leur territoire et d'autre part organisés en réseaux, via les mobilités des commerçants et les relations marchandes.

\section{Mobilités et logiques de réseaux}

Les acteurs, des colporteurs ruraux aux marchands urbains, construisent les ramifications discrètes par leurs mobilités et par leurs relations marchandes.

\section{Mobilités des commerçants}

Des mobilités ville-campagne, des mobilités à l'intérieur de l'espace rural, ou encore des mobilités entre la métropole régionale et la métropole nationale construisent des ramifications discrètes de la route transnationale. On distingue les mobilités portant sur des trajets à destination des lieux de vente ruraux, des mobilités concernant les déplacements vers les lieux d'approvisionnement urbain. Environ la moitié des vendeurs itinérants qui suivent le circuit des marchés périodiques ruraux est installée en ville (Bafoussam, Dschang), l'autre moitié réside dans des villages. 


\begin{tabular}{|l|l|l|l|l|l|l|l|}
\hline $\begin{array}{l}\text { Lieu } \\
\text { d'approvisionnement } \\
\text { LIEU DE VIE } \\
\begin{array}{l}\text { Ville } \\
\text { Village }\end{array}\end{array}$ & Lundi & Mardi & Mercredi & Jeudi & Vendredi & Samedi & Dimanche \\
\hline Chrétien & $\begin{array}{l}\text { Bafou } \\
\text { Samaya }\end{array}$ & DSCHANG & Mbelenka & Agriculture & Baleveng & $\begin{array}{l}\text { Fongo } \\
\text { Tongo }\end{array}$ & Mbelenka \\
\hline Odette & Dschang & Bansoa & Mbouda & Batcham & Baleveng & Bafoussam & BANGANG \\
\hline
\end{tabular}

Tableau 1 Deux parcours hebdomadaires de vendeurs itinérants

Ces deux parcours hebdomadaires sont intéressants pour montrer des itinéraires qui intègrent différemment la ville et la campagne. Chrétien commerce des sandales tous les jours. Il vit à Dschang et s'y approvisionne les mardis. Cinq autres jours, il vend dans des marchés ruraux dont deux fois à Mbelenka. Le jeudi, il cultive son champ. Odette vend de la layette, elle vit à Bangang et s'approvisionne à Bafoussam. Elle vend dans deux villes et quatre villages. Chrétien et Odette se retrouvent le vendredi à Baleveng mais ne se croisent pas les autres jours. Evidemment, ces deux profils n'épuisent pas la diversité des itinéraires hebdomadaires mais ils montrent que les marchés urbains et ruraux ont des places différentes dans les stratégies des marchands. Chrétien le citadin vend plus souvent dans des marchés ruraux tandis qu'Odette la villageoise passe trois journées dans des marchés urbains. Ce bref tableau révèle que la route de la pacotille imbrique différemment l'urbain et le rural dans les calendriers d'activités des marchands itinérants.

La densité importante des marchés ruraux offre une vaste gamme d'opportunités pour les marchands itinérants. Par conséquent, la diversité des parcours hebdomadaires est importante d'autant plus quand les marchands possèdent leur propre moyen de locomotion. Samuel et Frédéric sont originaires et vivent à Batcham, le premier vend des accessoires de mode et le second des sandales. Les deux possèdent une moto, ils écoulent leurs marchandises dans sept marchés chaque semaine mais ils n'ont pas le même parcours. La possession de leur propre véhicule augmente les possibilités et la liberté de choix de l'itinéraire.

Si des colporteurs aux plus faibles capitaux, et/ou en début de carrière, effectuent leur approvisionnement à Dschang ou à Mbouda, où des commerçants sont directement approvisionnés par les grossistes de Bafoussam, l'essentiel de l'approvisionnement par les colporteurs est effectué à Bafoussam et à Douala. Néanmoins, on trouve quelques commerçants de Bafoussam qui viennent directement approvisionner les petits colporteurs ruraux dans des marchés périodiques, telle Odile, rencontrée à Balessing. Elle vend en détail et en demi-gros aux «débrouillards », c'est-à-dire souvent les jeunes en début de carrière, « en partant [de Balessing], eux partent vendre, ils prennent pour 20000,30000 [Fcfa]» (30 à 45 euros). De jeunes vendeurs de Batcham sont aussi directement livrés par un commerçant originaire de Batcham qui s'approvisionne à Douala.

La moitié des marchands itinérants rencontrés sur les marchés périodiques, reconstitue le stock par des achats à Douala, parmi eux, la moitié fait le trajet une à deux fois par semaine, l'autre moitié l'accomplit une à deux fois par mois. Une faible part des vendeurs ambulants se rend à Douala moins d'une fois par mois. Il faut en principe environ $4 \mathrm{~h}$ pour rallier Douala depuis Dschang, 5 h30 depuis Bafoussam. Le voyage s'effectue en transport collectif dans des bus d'environ 16 ou 30 places ou dans une moindre mesure via des gros porteurs. La route de nuit est populaire, elle permet de faire un trajet sans perdre une demi-journée. Le développement du commerce bon marché est concomitant avec celui des transports, les mobilités profitent du développement des transports qui bénéficient de l'augmentation de la demande en mobilité. On 
note un phénomène cumulatif entre commerce itinérant et secteur des transports. Les trajets nombreux et réguliers vers Douala renforcent des relations entre les campagnes de l'Ouest et la métropole économique camerounaise.

Les déplacements des campagnes vers Bafoussam sont fréquents, la plupart des marchands ambulants qui se rendent dans la capitale régionale font le parcours une à deux fois par semaine. Les approvisionnements dans le comptoir régional et ceux dans le comptoir national sont complémentaires. On va plus facilement - en matière de capital et de temps - à Bafoussam et on va à Douala pour des volumes plus importants. Il n'est pas rare que les vendeurs modifient les sources d'approvisionnement en fonction du capital, du produit recherché et de la saison. Les vendeurs intègrent le coût financier du transport et le coût en matière de temps dans leurs activités. On observe des logiques de réseaux d'affinités qui nuancent la distance topographique et la contrainte de budget. Par exemple, des connexions avec un proche situé dans un comptoir éloigné - le réseau - peuvent remettre en cause la distance et court-circuiter le territoire. Les logiques de réseaux sociaux interfèrent avec les logiques de réseaux de lieux mais la substance de l'espace n'est pas gommée puisque la situation géographique est une donnée prise en compte.

En effet, l'accessibilité joue également un rôle important, d'autant plus dans des campagnes, certes où les pistes sont nombreuses mais où le relief doublé de l'absence de revêtement sont des contraintes, qui plus est lors de la saison des pluies. La proximité intervient. Par exemple, une part non négligeable de l'approvisionnement du marché rural de Batcham est réalisée via des marchands installés à Mbouda, distant de moins de $10 \mathrm{~km}$ à vol d'oiseau. Pour autant, la localisation du marché et la provenance du vendeur ne sont pas des critères suffisants pour établir des modèles de parcours entre lieux d'approvisionnement et lieux de vente, la variété et la labilité des parcours individuels rendent bien périlleuse toute velléité à établir un modèle. A Batcham, on peut rencontrer des vendeurs vivant à Batcham qui se ravitaillent très régulièrement à Mbouda, à Bafoussam, et d'autres qui vont à Douala une fois tous les trois mois. Par ailleurs, le marché rural de Baleveng, de Bangang, de Fongo Tongo, de Bafou Samaya et de Balessing, est directement connecté à Bafoussam et à Douala via les mobilités de petits entrepreneurs. On note tout de même que les vendeurs itinérants installés en ville (Dschang, Bafoussam) sont plus nombreux à se ravitailler à Douala que les colporteurs installés à la campagne. Ces circulations sans précédent recomposent ainsi les relations urbain-rural, ellesmêmes intégrées au réseau marchand global.

Par ailleurs, les rapports au temps sont également modifiés puisque l'essor de la route des pacotilles accélère le rythme du monde rural. En effet, la faiblesse du capital, la dépendance à la mode de certains produits et l'immédiateté des revenus sont des facteurs qui contribuent à hâter la fréquence des échanges. Les colporteurs ont souvent un capital financier et un stock limités. Par conséquent ils doivent s'approvisionner régulièrement et participent à l'intensification des circulations rural-urbain. Le rythme régulier est modifié par le facteur saisonnier. En effet, les échanges s'amplifient au moment de la rentrée scolaire et à la période des fêtes de Noël. Certains commerçants doublent le rythme d'approvisionnement à ces périodes. De plus, l'appartenance à une tontine - association d'épargne dont les bénéficiaires touchent les cotisations à tour de rôle - procure régulièrement un capital supplémentaire. Ce capital augmenté peut être réinvesti dans le stock et cela peut modifier la source d'approvisionnement. Douala sera privilégiée pour des volumes importants puisque les prix y 
sont légèrement inférieurs qu'à Bafoussam et que les marges de négociations y sont supérieures ainsi que le dit Tony « quand la tontine tombe, je m'approvisionne en gros à Douala ».

En outre, certains produits comme les vêtements se démodent rapidement et deviennent invendables. Cela signifie que les marchands doivent être informés des dernières nouveautés dans un secteur qui est très concurrentiel en ville et à la campagne. Joseph, vendeur itinérant de vêtements, installé à Dschang, souligne le fort renouvellement des produits et le caractère volatile du secteur quand il affirme que « le marché c'est la loterie, c'est des trucs qui sortent en nouveauté tous les jours, il faut aller tenter le marché avec la nouveauté ». Aristide, vendeur de bijoux fantaisie à Bafoussam ne déclare-t-il pas «c'est le siècle de vitesse, si tu ne fais pas comme ça tu rentres au village »? Il oppose finalement le temps de la ville, celui du business urbain, avec le temps des campagnes, celui des saisons agricoles, lent et prévisible. Néanmoins, ce temps des campagnes est aussi en train de gagner en vitesse via le développement des connexions à la ville et à la globalité. Au total, avec l'essor du commerce bon marché, c'est le rapport au temps qui est bouleversé. Les revenus agricoles saisonniers et les revenus hebdomadaires ou mensuels du salariat peuvent être perçus, particulièrement par la jeunesse, comme archaïques, alors que l'immédiateté des revenus est positivement perçue, fussent-ils modiques. L'essentiel, c'est peut-être l'échange libéré de l'attente. La route des pacotilles chinoises contribue à des rapports à l'espace et au temps inédits, à des modes de vie basés sur la circulation, sur la flexibilité et sur l'immédiateté.

Marchand itinérant : une profession en lien avec l'agriculture, ancrée dans des logiques de réseaux multiples

Une petite majorité de marchands itinérants sont des hommes. Au moment des entretiens en 2014, la moitié des commerçants exerce ce métier depuis plus de dix années. Cela montre que le commerce de pacotille est une source durable de revenus et que des carrières dans ce domaine sont possibles. Le capital initial provient d'activités antérieures dans la construction, le commerce de produits de brousse, l'artisanat, les services, etc. et pour une part importante de l'agriculture. Le secteur agricole est important dans les campagnes de l'Ouest, il procure des revenus issus de la vente de tout ou partie de récoltes mais il fournit aussi des emplois, notamment de tâcheron. La majorité des colporteurs a débuté le commerce des pacotilles avec un capital compris entre 10000 et 50000 Fcfa (15 à 75 euros), certains ont démarré avec 3000 Fcfa (4,6 euros), d'autres avec rien. Ces derniers ont pu accéder au commerce et apprendre le métier en tant que vendeur assistant, souvent auprès d'un membre de la famille. Il est possible de bénéficier de marchandises à crédit, en particulier pendant les vacances scolaires pour une horde de jeunes. En effet, les écoliers sont poussés à fournir une contribution financière, notamment pour les frais de scolarité de la rentrée suivante. Lors des grandes vacances, ils sont très nombreux à vendre quelques articles manufacturés bon marché fournis à crédit par un proche ou des produits issus du champ. Les plus débrouillards peuvent accumuler jusqu'à 100000 Fcfa (150 euros) en trois mois. Ces expériences nourrissent des compétences professionnelles voire suscitent des vocations ou deviennent la première étape professionnelle par défaut pour ceux qui ne poursuivront pas leur scolarité, souvent faute de capacité financière du ménage. Le commerce bon marché est aussi une opportunité pour tenter d'accumuler du capital, voire même du capital foncier. Les carrières sont à étapes, de nombreux commerçants sédentaires au marché A ont démarré par la vente de rue «informelle », à l'exemple d'Eric, 
ancien vendeur de rue et désormais grossiste en vêtements, " je me suis battu moi-même, je faisais d'abord la sauvette, c'est à partir de la sauvette que je me suis lancé ». La boutique en ville demeure l'horizon de très nombreux vendeurs itinérants.

Plus des deux tiers des marchands rencontrés dans les campagnes (y compris ceux qui vivent en ville) ont accès à des ressources agricoles, soit directement parce qu'ils cultivent eux-mêmes, soit indirectement parce qu'au moins un membre du ménage cultive, en particulier le ou la conjointe. Pour ceux qui cultivent, le travail au champ se combine avec la vente itinérante dans le calendrier hebdomadaire. Ce dernier est modifié en fonction des saisons puisque certaines tâches agricoles requièrent plus de force de travail. De plus, les revenus issus d'une activité peuvent bénéficier à l'autre activité. Le capital circule entre le commerce bon marché et l'agriculture, dans les deux sens, à des rythmes qui suivent le calendrier agricole et celui du marché des pacotilles (cf. rentrée scolaire et Noël). Il existe donc une complémentarité financière entre les deux activités. Pour les ménages qui cultivent sur un hectare - surface moyenne -, aucune des deux activités ne dominent l'autre sur le plan pécuniaire, « avec les deux on jongle, il n'y a pas de côté fort » (Chrétien). La multi-activité apparaît nécessaire ainsi que le déclare Boniface, installé à Dschang, «si tu fais seulement le commerce, tu ne vas pas t'en sortir ». Il cultive deux fois par semaine et vend quatre jours par semaine dans quatre marchés différents (Bafou Samaya, Dschang, Mbelenka et Fongo Tongo). De plus, même des grossistes du marché A indiquent que l'agriculture peut jouer le rôle d'amortisseur de crise, par exemple Eric : «l'agriculture a sa place, la majorité des commerçants font le champ parce qu'on ne sait jamais, si ça ne peut pas donner, tu rentres au champ ».

L'écrasante majorité des marchands itinérants est originaire de l'Ouest. Le réseau social, à affinités communautaires doublées de convergences d'intérêts professionnels joue un rôle déterminant. Le soutien financier, sous diverses formes, et l'introduction par un proche au réseau des pacotilles sont fréquents. L'appui monétaire provient d'un prêt ou d'un don de la famille proche, ou de la tontine - indépendamment de l'attribution cyclique des cotisations. Le soutien c'est également l'information, indispensable pour bien démarrer et pour découvrir rapidement les secrets des affaires : informations diverses portant sur les contacts, les prix, les produits, les lieux et le fonctionnement. Il n'est pas rare que le novice, d'autant plus s'il est d'extraction paysanne et non familier avec la ville, fût-elle à quelques dizaines de kilomètres, soit accompagné par un proche qui joue alors le rôle de guide, voire de facilitateur pour la première prise de contact avec les commerçants urbains. Ghislaine ne dit-elle pas «il faut un entraineur »? Pour autant, une minorité de marchands déclarent n'avoir bénéficié d'aucun soutien.

Le réseau social est aussi utile en cas de coups durs, il est un élément de résilience de commerçants (Berrou et Gondard-Delcroi 2011). Les multiples associations (tontines, comités de développement, groupes de commerçants, groupes religieux, syndicats, etc.) s'avèrent de relatifs amortisseurs de crise mais l'appartenance induit une capacité de cotisation et la possibilité/volonté à respecter les engagements qui lient aux différents groupes. Ces obligations financières et sociales découragent des marchands, en particulier ceux qui ne sont pas capables d'épargner ou de cotiser. Les motifs d'association reposent sur différents facteurs, par exemple l'origine géographique, la localisation de l'activité, le type de produits, etc. Des associations recoupent les critères de regroupement, par exemple le groupe des jeunes vendeurs de bijoux fantaisie de Batcham. L'appartenance multiple permet à des membres d'utiliser leurs positions multiples pour des intérêts spécifiques, elle est gage de succès plus importants que l'isolement 
de certains marchands (Walther, 2015). Ces appartenances socio-professionnelles sont aussi un moyen d'intégration urbain-rural. Eric, grossiste en vêtements, est membre d'au moins un groupe de grossistes au marché $\mathrm{A}$ et d'une tontine - réunion - aux alentours de Mbouda, d'où il est originaire.

La vente à crédit est un moyen très répandu pour créer des relations non éphémères voire durables, et pour capter le client. Les grossistes incitent leurs clients à prendre en général entre un quart à un tiers de la marchandise à crédit. Cette pratique est nécessaire pour les échanges ainsi que le déclare Eric, «les avances sont obligatoires pour fonctionner ». Le crédit est pratiqué entre les importateurs et les grossistes et entre les grossistes et les marchands itinérants. Il repose sur la confiance, celle-ci s'obtient si les échanges sont réguliers, éventuellement à partir de quatre transactions. Dans tous les cas, le créancier exige les coordonnées du créditeur. Cette pratique est plus vive lors des hautes saisons commerciales pour fluidifier les circulations. Elle entre dans un cadre très concurrentiel, l'offre et la demande sont élevées. Bien que des marchands itinérants refusent, à l'image de Sam, pourtant vendeur chevronné avec huit ans d'expérience « le crédit n'est pas bien, ça a des risques, il [le grossiste] veut déjà me noyer » (Sam), le crédit est un moyen d'établir des relations relativement durables entre acteurs. En ce sens, le crédit est un levier de construction de la route des pacotilles.

Les réseaux mystiques font également partie du monde de la pacotille chinoise dans l'Ouest du Cameroun. La dimension spirituelle est présente dans des représentations qui influencent les pratiques ${ }^{15}$. Il y aurait un stade à partir duquel les réseaux mystiques de parrainage joueraient un rôle déterminant. Cela concernerait par exemple l'accès à une grosse somme d'argent, une quantité importante de marchandises à un prix défiant toute concurrence, ou l'accès à l'information stratégique, comme celle portant sur l'accès à la source directe en Chine ou à Dubaï. Des «parrains » proposeraient ces services, or, cela induirait un pacte implicite, une compromission. La question de l'acceptation du pacte est très ambigüe, la partie bénéficiaire peut consentir en toute connaissance de cause ou dans d'autres cas à son insu. Ces parrainages expliqueraient des succès fulgurants, précédant des chutes brutales, ainsi que l'atteste l'adage « vivre heureux et mourir jeune ». Les conditions du pacte seraient rarement connues, la contrepartie peut être par exemple le don d'un proche en sacrifice. Dès lors que le pacte, consenti ou pas, est validé, impossible de faire machine arrière «non, tu as déjà consommé au point où tu es ». Joseph, qui est par ailleurs commerçant assure que " c'est très difficile de s'engager quand on ne connaît pas la personne qui se propose ». Pourtant, il déclare aussi quand on évoque ses projets "grossir, mais c'est l'argent qui me manque pour pouvoir partir en Chine ». Pour Joseph, la crainte de la compromission est un frein dans la stratégie de capitalisation de l'activité. Quoi que puisse être le regard sur la dimension mystique, les acteurs du secteur des produits importés intègrent le monde spirituel dans les stratégies commerciales. Autrement dit, les ramifications discrètes de la route des pacotilles se dessinent aussi via des logiques culturellement ancrées, fussent-elles inavouables, inadmissibles ou hors du champ du fameux homo oeconomicus ${ }^{16}$.

\footnotetext{
15 Informations recueillies dans des conditions spécifiques : préparation minutieuse, accompagnement, lieu favorable (salle privée dans un bar). Informations recoupées par deux universitaires originaires de l'Ouest.

${ }^{16}$ L'homo oeconomicus est à la base de l'analyse économique néoclassique. L'homo oeconomicus est un individu universel, il effectue des choix « rationnels » en vue d'obtenir l'utilité la plus élevée possible, il cherche la maximalisation de sa satisfaction en utilisant au mieux ses ressources. Ses choix sont départis d'éléments psychologiques, des particularités sociales, culturelles, historiques, et géographiques des sociétés. Notion
} 


\section{Conclusion}

La circulation des pacotilles chinoises relie des marges rurales et des villes secondaires à des routes marchandes transnationales. La partie aval de cette route transnationale est composée de ramifications discrètes entre des villages de l'Ouest camerounais, des villes secondaires et une métropole régionale. L'approche par les acteurs et par les lieux du quotidien met en lumière comment des marges rurales et des villes secondaires s'intègrent à l'économie mondialisée via la circulation de la pacotille chinoise et comment ce mouvement contribue à des relations ruralurbain sans précédent. Le marché A à Bafoussam est une double interface, d'une part entre l'agriculture locale et le marché national et sous-régional, d'autre part entre des produits importés de Chine et les consommateurs urbains et ruraux. La structuration du marché A et son développement sont en partie le résultat du développement du secteur des produits importés de Chine. Le Marché A joue un rôle structurant, avec des connexions directes aux comptoirs asiatiques et moyen-orientaux, dans l'intégration de la ville et de son arrière-pays rural et urbain au réseau transnational. Les marchés ruraux, lieux principaux de pénétration des marchandises importées dans les campagnes illustrent le fait que l'essor de la route des pacotilles repose sur le dynamisme de l'agriculture locale.

L'ampleur inédite des mobilités liées au commerce bon marché intensifie les relations urbainrural par les nombreux trajets destinés à l'écoulement des produits et par les déplacements dans les comptoirs d'approvisionnement. Ces connexions entre des lieux via les mobilités des marchands construisent des ramifications discrètes de la route transnationale. La circulation accrue élabore des rapports inédits à l'espace et au temps, des modes de vie basés sur la flexibilité et sur l'immédiateté. L'exposition plus fréquente aux vitrines et aux objets de la globalisation participent à des identités sans précédent qui intègrent plus facilement la ville et la campagne, le local et "l'ailleurs ». Les jeunes sont très perméables à ces changements puisque le temps et l'espace des produits importés jouissent d'une image positive, qui plus est dans des sociétés où les perspectives d'accumulation via l'agriculture induisent un capital financier et foncier inaccessible pour une grande partie d'entre eux. L'immédiateté des revenus et le fait d'être en prise avec la mode, sont en rupture avec des représentations du village perçu parfois comme le monde des contraintes sociales, ennuyeux et sans promesse d'horizon faste. Les trajectoires professionnelles sont marquées par des étapes et bénéficient fréquemment d'obligations sociales, en particulier des ainés sur les cadets. Elles s'appuient également sur des solidarités de groupe, rarement déconnectées de logiques communautaires. Ces solidarités sont aussi des moteurs locaux de structuration de la route des pacotilles.

Le local, la périphérie rurale et le réseau urbain secondaire jouent un rôle fondamental dans la structuration de ces routes globales, ces dernières tirant parti du contexte macroéconomique favorable pour les échanges sino-camerounais. Les acteurs privés, en particulier du bas, colporteurs ruraux, commerçants urbains, etc. sont les moteurs de ces ramifications discrètes. Cela questionne la maîtrise de l'espace par l'Etat. Ces logiques du monde privé, qui d'ailleurs élabore souvent des stratégies pour échapper à l'Etat, sont-elles une réponse au constat d'échec du rôle de l'Etat? L'essor du commerce bon marché est-il une continuité du développement du vivrier marchand, une autre démarche de contournement voire de subversion du système ? Les

contestée, voir Rist G. 2010, L'économie ordinaire entre songes et mensonge, Paris, Les Presses de Sciences Po, $250 \mathrm{p}$. 
acteurs du bas et ces marges transforment bien un contexte socio-économique et politique en opportunités. Evidemment, ce n'est pas la panacée, le commerce de pacotille est-il un levier de développement, aura-t-il des effets positifs d'entrainement ? Henri, vendeur itinérant chevronné de Batcham, nuance l'engouement pour ce secteur, dont les réussites individuelles sont de plus en plus remises en cause par l'augmentation de la concurrence, « ça ne marche pas bien, mais je force à faire cela », faute de mieux pourrait-on ajouter. D'ailleurs, le commerce de la pacotille ne tourne pas le dos à l'agriculture puisque ces deux activités se combinent dans le calendrier hebdomadaire et saisonnier et dans la circulation du capital.

Les effets de contournement s'observent par ailleurs dans l'espace entre les lieux. Des logiques de réseau dépassent le continuum urbain-rural puisqu'on a montré des court-circuitages à travers par exemple des connexions directes entre un village et Douala, sans que Bafoussam bénéficiât de sa position. On a aussi relevé que des importateurs discrets de Bafoussam contournent le marché de Douala, leurs containers ne faisant qu'y transiter. Or, la route des pacotilles combine ces court-circuitages du continuum urbain rural avec le renforcement des relations - e.g. mobilités - dans le continuum urbain-rural.

Finalement, ces ramifications discrètes de la route des pacotilles rendent intelligibles une part de la mondialisation et des relations urbain-rural. La partie informe sur le tout qui informe sur la partie. Les dynamiques spatiales permettent de lire localement la route globale. Cela suppose que la connaissance du lieu, des moteurs et des acteurs qui participent à sa construction, permet de comprendre la dynamique du réseau.

\section{Bibliographie}

Agergaard J., Fold N., Gough K. (éd.), 2009 - Rural Urban Dynamics: Livelihoods, Mobility and Markets in African and Asian Frontiers. Londres et New York : Routledge, 232 p.

Belguidoum S., Pliez O., 2015 - "Yiwu: The creation of a global market town in China", Articulo - Journal of Urban Research $\mathrm{n}^{\circ} 12$. DOI : 10.4000/articulo.2863

Berrou J-P., Gondard-Delcroi C., 2011 - «Dynamique des réseaux sociaux et résilience socio-économique des micro-entrepreneurs informels en milieu urbain africain », Mondes en développement $\mathrm{n}^{\circ} 156$. DOI : https://doi.org/10.3917/med.156.0073

Bertoncello B., Bredeloup S., 2007 - «De Hong Kong à Guangzhou, de nouveaux “comptoirs" africains s’organisent », Perspectives chinoises $\mathrm{n}^{\circ} 1$, p. 98-110.

Bromley R., 2000 - "Street vending and public policy: a global review", International Journal of Sociology and Social Policy n²0 (1/2), p. 1-28.

Bryceson D.F., 1999 - "African rural labour, income diversification \& livelihood approaches: A long-term development perspective". Review of African Political Economy, vol. 26, n 80, p. 171-189. DOI : https://doi.org/10.1080/03056249908704377

Cabestan J.-P., 2015 - "China-Cameroon relations: Fortunes and limits of an old political complicity", South African Journal of International Affairs n²2(1). DOI : https://doi.org/10.1080/10220461.2015.1014930

Chaléard J.-L., 1996 - Temps des Villes, Temps des Vivres. L'Essor du Vivrier Marchand en Côte d'Ivoire. Paris : Karthala, 660 p.

Chaleard J.-L., Dubresson A. (éds), 1999 - Villes et campagnes dans les pays du Sud. Paris : Karthala, $258 \mathrm{p}$.

Charlery de la Masselière B., 2013 - « Systèmes spatiaux, systèmes de ressources et identités : redéfinir les contextes des dynamiques territoriales ». In : B. Charlery de la Masselière, 
B. Thibaud et V. Duvat (dir.), Dynamiques rurales dans les pays du Sud, l'enjeu territorial. Toulouse : Presses universitaires du Mirail, p. 9-20.

Chen M. A., 2012 - "The Informal Economy: Definitions, Theories and Policies, Women in Informal Employment: Globalizing and Organizing", WIEGO working paper ${ }^{\circ} 1$. Cambridge, WIEGO, https://www.wiego.org/publications/informal-economy-definitions-theories-andpolicies (Consulté le 26 juillet 2019)

Choplin A. et Pliez O., 2015 - “The Inconspicuous Spaces of Globalization”. Articulo, n 15, https://journals.openedition.org/articulo/2905 (Consulté le 26 juillet 2019)

Doron A, 2015 - «De la marge au monde : la structuration mouvementée d'une place marchande transnationale à Ben Gardane (Tunisie) », Les Cahiers d'EMAM n²6. DOI: 10.4000/eps.7170

Ela J.-M., 1998 - Innovations sociales et renaissance de l'Afrique noire. Paris : L'Harmattan, 426p.

Gabas J.-J., Chaponnière J.-R., 2012 - Le temps de la Chine en Afrique. Paris : Karthala, 2017 p.

Gereffi G., Korzeniewicz M. (ed.), 1994 - Commodity Chains and Global Capitalism. Westport : Greenwood Press, 332 p.

Gereffi G., Humphrey J., Sturgeon T., 2005 - "The governance of Global Value Chains", Review International Political Economy n ${ }^{\circ} 12-1$, p. 78-1004.

Institut National de la Statistique, 2015 - Annuaire statistique du Cameroun, Edition 2015, Yaoundé, Institut National de la Statistique, 536 p.

Kernen A., Khan Mohammad G., 2014 - «La révolution des produits chinois en afrique consommation de masse et nouvelle culture matérielle », Politique africaine $\mathrm{n}^{\circ} 134$, p. 111-132.

Lesourd M., 1997 - « L'archipel rural africain en mouvement ». In : J.-Y. Gastellu, La ruralité dans les pays du Sud à la fin du XXe siècle. Paris : ORSTOM, p. 363-380.

Mainet H., Racaud S., 2015 - « Secondary towns in globalization: Lessons from East Africa », Articulo - Journal of Urban Research $n^{\circ}$ 12. DOI: 10.1080/00167223.2017.1354715

Marcus G.E., 1995 - "Ethnography in/of the world system: the emergence of multi-situated Ethnography". Annual Review of Anthropology n²4, p. 95-117.

Massey D., 1991 - “A global sense of place”, Marxism Today n³8, p. 24-29.

Morin E., 1977 - La Méthode, 1. La nature de la nature, Paris, Seuil, 400 p.

Nokam M.N., 2012 - Approvisionnement et gestion des espaces marchands de vivres à Bafoussam. Dschang, Université de Dschang, mémoire de Master, 215 p.

Pélissier P., 2000 - Les interactions rural-urbain: circulation et mobilisation des ressources. Bulletin de l'APAD n¹9, URL : http://apad.revues.org/422

Pezzano A., 2016 - “'Integration' or 'selective incorporation'? The modes of governance in informal trading policy in the inner city of Johannesburg", The Journal of Development Studies n 52 (4), p. 498-513.

Pliez O., 2007 - Des jeans chinois dans les rues du Caire, ou les espaces discret de la mondialisation. Mappemonde $\mathrm{n}^{\circ} 88$ http://mappemonde.mgm.fr/num16/articles/art07404.html (Consulté le 3 septembre 2019).

Racaud S., 2018 - « Ambiguous Resource: "Informal" Street Trading in Kisumu, Kenya », Articulo - Journal of Urban Research n ${ }^{\circ} 7-18$. DOI : 10.4000/articulo.3702 
Racaud S., Kago J., Owuor S., 2018 - «Introduction: Contested Street: Informal Street Vending and its Contradictions », Articulo - Journal of Urban Research $n^{\circ} 17-18$ URL : http://journals.openedition.org/articulo/3719 (Consulté le 3 septembre 2019).

Roever S., Skinner C., 2016 - "Street vendors and cities", Environment and Urbanization $\mathrm{n}^{\circ}$ 28(2), http://wiego.org/publications/street-vendors-and-cities (Consulté le 3 septembre 2019).

Tacoli C., 1998 - "Rurban-urban interactions : a guide to the literature",Environment and Urbanization, Vol. 10, No 1, p. 147-166.

Tacoli, C. (éd.), 2006 - The Earthscan reader in rural-urban linkages. Londres et New York : Earthscan Routledge, $350 \mathrm{p}$.

UN-Habitat, 2015 - Habitat III issue papers 10 - Urban-rural linkages. New-York, 9 p.

Tarrius A., 2002 - L amondialisation par le bas : les nouveaux nomades de l'économie soutarraine. Paris, Balland, 169 p.

Walther O., 2015 - "Business, brokers and borders: The structure of West African trade networks". Journal of Development Studies n51 (2), p. 603-620.

Yemmafouo A., 2013 - Urbanisation et espaces périurbains en Afrique subsaharienne. Pratiques à l'Ouest-Cameroun. Paris : L'Harmattan 270 p.

Yemmafouo A., 2018 - Street Vending Power Relationships and Governance of Public Spaces in Bafoussam, West Cameroon, Current Urban Studies $\mathrm{n}^{\circ} 6$, https://doi.org/10.4236/cus.2018.64032 